\title{
A geometric acoustics approach to the study of sound propagation in ducts containing sheared flows
}

\author{
Denny W. Grimm \\ Procter and Gamble Company, Cincinnati, Ohio 45224
}

C. J. Hurst

Mechanical Engineering Department, Virginia Polytechnic Institute and State University, Blacksburg, Virginia 24061

(Received 6 November 1978; accepted for publication 15 August 1979)

\begin{abstract}
Geometric acoustics has been used to study the propagation of sound waves in a homogeneous moving medium with sheared flow bounded by the hard walls of a duct. Differential equations describing the ray trajectories and the spreading losses along each ray were developed and solved numerically for a range of centerline Mach numbers and shear boundary-layer thicknesses. Results were obtained which show the effects of upstream and downstream sound propagation on the ray paths. A method was also developed to allow the calculation of intensity loss profiles at specified downstream cross sections of the duct.
\end{abstract}

PACS numbers: 43.20.Dk, 43.20.Mv

\section{INTRODUCTION}

The transmission of sound through ducts and tubes is a subject which has attracted considerable attention recently because of its importance in the control of jet engine noise. The solution to the problem of sound transmission through a duct of constant cross-sectional area without fluid flow is well known. However, the situation becomes more complex when fluid flow through the duct is included. Further complication is added if the crosssectional area of the duct is allowed to vary.

Numerous attempts have been made to solve the differential wave equations for transmission through a duct carrying flow. The case of a duct with a uniform flow has been treated by Ingard, ${ }^{1}$ Eversman, ${ }^{2}$ Morfey, ${ }^{3}$ Tack and Lambert, ${ }^{4}$ and Doak and Vaidya, ${ }^{5}$ among others. Pridmore-Brown ${ }^{6}$ was the first to solve the wave equation with the inclusion of both uniform flow and flowgradient effects for sound propagating between two plane parallel walls. Pridmore-Brown solved this equation for downstream propagation of the lowest order mode in the shear layer for both constant gradient and oneseventh power-law flow profiles, assuming an inviscid fluid. His solution for downstream propagation predicted an increased sound-pressure level near the hard walls caused by refraction of the sound wave by the sheared flow. The effects of refraction were found to be more pronounced at higher frequencies and higher Mach numbers. The solution obtained by PridmoreBrown, however, did not hold near the duct walls for the turbulent flow case. The difficulties near the wall were overcome in a numerical solution obtained by Mungur and Gladwell. ${ }^{7}$ Their results agreed quite well with Pridmore-Brown's solution at points not near the duct wall and was valid at points near the duct wall as well. The effects of a flowing medium have also been confirmed experimentally in work performed by Mechel, Mertens, and Shilz. ${ }^{8}$ For downstream propagation, they found a frequency below which attentuation decreased with flow and above which attenuation increased with flow. For upstream propagation, convection effects caused increased attenuation and refraction effects caused decreased attenuation. Mungur and Plumble ${ }^{9}$ modeled the flow in a cylindrical duct with a constant velocity core and a shear layer near the walls. Their objective was to study the effect of varying the shearlayer thickness. For downstream propagation the effects of sound refraction were found to increase with increasing boundary-layer thickness and were found to cause higher sound pressure levels near the duct walls. Eversman $^{10}$ found, however, that this refraction of sound for downstream propagation was less significant than for upstream propagation. Eversman also showed a pronounced decrease in acoustic wall liner performance due to upstream refraction. Hersh, Beranek, and Newman ${ }^{11}$ concluded that the effects of shear-layer refraction became significant when the ratio of shearlayer thickness to acoustic wavelength was equal to or greater than unity. In yet another study on boundarylayer refraction, Savkar ${ }^{12}$ also concluded that refraction effects cannot be ignored for the high Mach numbers and high frequencies typically encountered in turbojet engines.

The problems of finding normal-mode solutions to the wave equations for sound being transmitted in a duct with flow, and possibly with variable cross-sectional area, remain unsolved. A review paper by Nayfeh, Kaiser, and Telionis ${ }^{13}$ indicates the considerable amount of effort that has been applied to finding a solution. The best approaches at present seem to be numerical in nature and are based on various approximations. It seems appropriate, the refore, to consider an approximate solution to the problems in terms of geometric acoustics. Despite low-frequency limitations,${ }^{14}$ the method has the advantage of simplicity and gives a clear picture of the sound field.

\section{MODEL TO BE STUDIED}

The model investigated in this study was a two-dimensional one. That is, the flow and sound propagation were assumed to occur between two parallel planes of infinite length and width. These "duct" walls were as- 


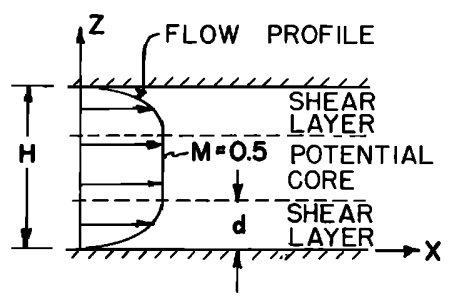

FIG. 1. Two-dimensional duct model. Flow profile is for a centerline Mach number of 0.5 and a shearlayer thickness of $30 \%$ of the duct height.

sumed to be perfectly rigid and smooth. The temperature in the duct was assumed to be uniform and all viscous absorption by the medium was neglected due to the short propagation distances considered.

The flow model used consisted of a potential core in the duct center matched to a boundary layer of constant thickness near the duct walls. The model used and the coordinate system chosen are illustrated in Fig. 1. This model was felt to be a realistic one for those cases where turbulent flow exists but is not fully developed, such as found in aircraft engine inlet and exhaust ducts. The flow model was similar to one used by Mungar and Plumblee, ${ }^{15}$ and by Hersh and Catton, ${ }^{16}$ except that these investigators used a quarter of a sine wave to model the flow profile in the boundary layer.

Considerable thought was given to the choice of an appropriate flow model in the boundary layer for this investigation, since the simple models used by previous investigators would not work for the geometric acoustics study. In geometric acoustic calculations in a flow field, the curvature of the sound rays is dependent upon the gradient of the flow field, and the spreading of the rays is dependent on the spatial changes in the flow gradient. For these reasons it was necessary that the first and second spatial derivatives of the flow field be defined and continuous functions. The equation for the shear layer must have first and second spatial derivatives which vanish at the interface between the potential core and the boundary layer, and which remain finite at points very near the duct wall. A power-law flow model was developed which fulfilled these requirements. The equation for this flow profile was

$$
M(z)=M_{\mathrm{cl}}\left\{1-\left[(d)^{-m}(d-z)^{m}\right]\right\},
$$

where $M(z)$ is the Mach number at height $z, M_{\mathrm{cl}}$ is the centerline Mach number, $d$ is the thickness of the shear layer, and $m$ is an exponent chosen to obtain a realistic profile. This equation was used to establish the flow parameters in the lower shear layer $(0 \leqslant z \leqslant d)$, and symmetry was used to establish the flow parameters in the upper shear layer. Various flow profiles were considered by specifying the centerline Mach number $M_{\mathrm{c} 1}$ and the boundary-layer thickness $d$. By experimentation, a realistic profile was achieved by using an integer power $m$ of four. The profile obtained compared favorably with those used by previous investigators, and with the seventh power flow profiles often discussed in fluid mechanics literature.

In accordance with the two-dimensional approach to this problem, the sound source was assumed to be a pulsating line source parallel to the $y$ axis.

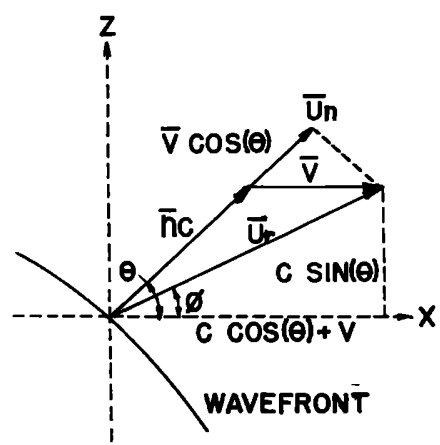

FIG. 2. Phase velocity and ray velocity directions in a moving medium.

\section{RAY TRACING EQUATIONS}

In the presence of a sheared flow, acoustic rays do not remain straight but are bent in the $x-z$ plane because of diffraction effects. A set of coupled nonlinear differential equations must be solved to find the ray paths through the moving medium. These paths represent the trajectories along which the acoustic energy of the sound wave is flowing. The derivation of these equations for the ray paths will be presented next.

Figure 2 shows a wave travelling through a moving medium. The wavefront is shown at an arbitrary time $t$, proceeding through the medium in the direction given by the wavefront normal vector $n$. The direction of the wavefront normal vector is given by the angle $\theta$. The medium through which the sound is travelling is assumed to have a velocity $\mathbf{V}$ which is a function of $z$ and which is parallel to the $x$ axis.

As discussed in Hayes, Haefeli, and Kulsrud, ${ }^{17}$ an acoustic ray can be considered as the trajectory of a point which moves from the point of emission of a spherelet to the point of tangency of the spherelet with the envelope wavefront at a time $\Delta t$ later. This is shown in Fig. 3, where the wavefront is shown at $t_{0}+\Delta t$ tangent to spherelets which originated at the initial wavefront. The position vector $r$ shows the location of the ray point at time $t+\Delta t$. This point moves with the group velocity $\mathrm{U}_{r}$

$$
\mathbf{U}_{r}=\frac{d \mathbf{r}}{d t}=c \mathbf{n}+\mathbf{V} .
$$

The $\mathbf{U}_{r}$ vector is shown in Fig. 2. It is evident that this vector is not generally perpendicular to the wavefront, but is inclined at angle $\phi$.

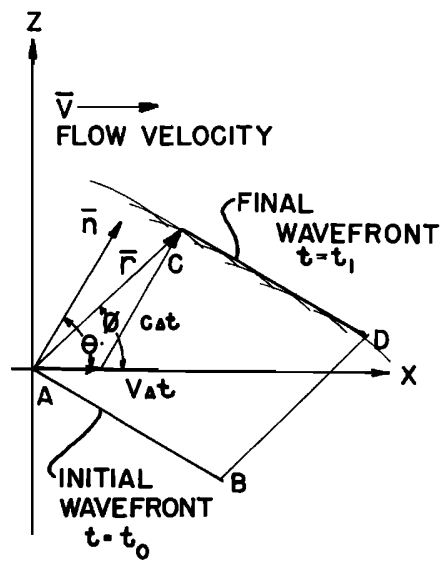

FIG. 3. Wave propagating through constant flow velocity region. 
The group velocity $\left(U_{r}\right)$ is different from the phase velocity $\mathrm{U}_{n}$. The phase velocity is the velocity with which the wave expands normal to one wavefront. The direction of the wave normal $\mathbf{n}$ is given by $\theta$, shown on Fig. 2. The phase velocity is given by

$$
\mathbf{U}_{r}=(c+\mathbf{n} \cdot \mathbf{V}) \mathbf{n} \text {. }
$$

The determination of the ray trajectory is obtained by integration of the group velocity equation. This is most easily done in this case in terms of its coordinate components. As Fig. 2 makes evident,

$$
\begin{aligned}
& \frac{d x}{d t}=\left(\mathrm{U}_{r}\right)_{x}=c \cos \theta+V, \\
& \frac{d z}{d t}=\left(\mathrm{U}_{r}\right)_{z}=c \sin \theta .
\end{aligned}
$$

Along with these equations it is also necessary to know how the wave normal vector $n$ changes along the ray path. The refraction law for the wave normal in its most general form, can be written as (see, for example, Hayes et al. ${ }^{17}$ )

$$
\frac{-d \mathbf{n}}{d t}=\nabla c+(\nabla \mathbf{V}) \cdot \mathbf{n}-\mathbf{n}[\mathbf{n} \cdot \nabla c+\mathbf{n} \cdot(\nabla \mathbf{V}) \cdot \mathbf{n}] .
$$

In this study the speed of sound was assumed constant across the duct, and the flow velocity was a function of $z$ only. The unit normal can be replaced by its components. Under these conditions, Eq. (5) reduces to

$$
\frac{d \theta}{d t}=-\cos ^{2} \theta \frac{d V(z)}{d z} .
$$

The solutions for $x, z$, and $\theta$, from Eqs. (4a), (4b), and (6), completely describe the paths of the sound rays through a boundless medium.

The medium under study is bounded, however, by the wall of the duct. At those surfaces the assumption was made of total reflection of the ray with no phase change. Therefore, the only change in the ray-trace variables was a sign change for the ray-normal angle since the angle of reflection necessarily equalled the angle of incidence. Thus the boundary conditions at the walls are

$$
\Delta x=0, \Delta z=0, \Delta \theta=-2 \theta .
$$

Each ray path can then be uniquely defined by specifying an initial source position $\left(x_{0}, z_{0}\right)$ and wave normal angle $\theta_{0}$.

To make the results of this work more generally useful, the following nondimensional parameters were used:

$$
\begin{aligned}
& M=V / c, \text { the Mach number, } \\
& T^{*}=t c / H, \text { nondimensional time, } \\
& L^{*}=L / H \text { nondimensional duct length, } \\
& x^{*}=x / H \text { nondimensional } x \text { coordinate, } \\
& z^{*}=z / H \text { nondimensional } z \text { coordinate }, \\
& F^{*}=f H / c \text { nondimensional frequency, also equal to } \\
& \quad \text { the duct height divided by the wavelength, } \\
& c=\text { speed of sound } .
\end{aligned}
$$

With these definitions, the nondimensional ray trace equations solved were:

$$
\begin{aligned}
& \frac{d x^{*}}{d T^{*}}=\cos \theta+M\left(z^{*}\right), \\
& \frac{d z^{*}}{d T^{*}}=\sin \theta, \\
& \frac{d \theta}{d T^{*}}=-\cos ^{2} \theta \frac{d M\left(z^{*}\right)}{d z^{*}} .
\end{aligned}
$$

\section{RAY TRACING RESULTS}

The solutions to the ray-trace equations were found numerically through the use of a Runge-Kutta integration routine. The computer output was generated in graphical format. The result acoustic ray diagrams are shown in Figs. 4-7.

Figures 4 and 5 show the sound rays for upstream propagation (i.e., for the sound propagating against the flow). Both are for a boundary-layer thickness equal to $30 \%$ of the duct height. The centerline Mach number is the only difference between the conditions for these two figures. In the first case, a centerline Mach number of 0.5 was used, while the Mach number of 0.7 was used to generate the second diagram. The diffraction of the

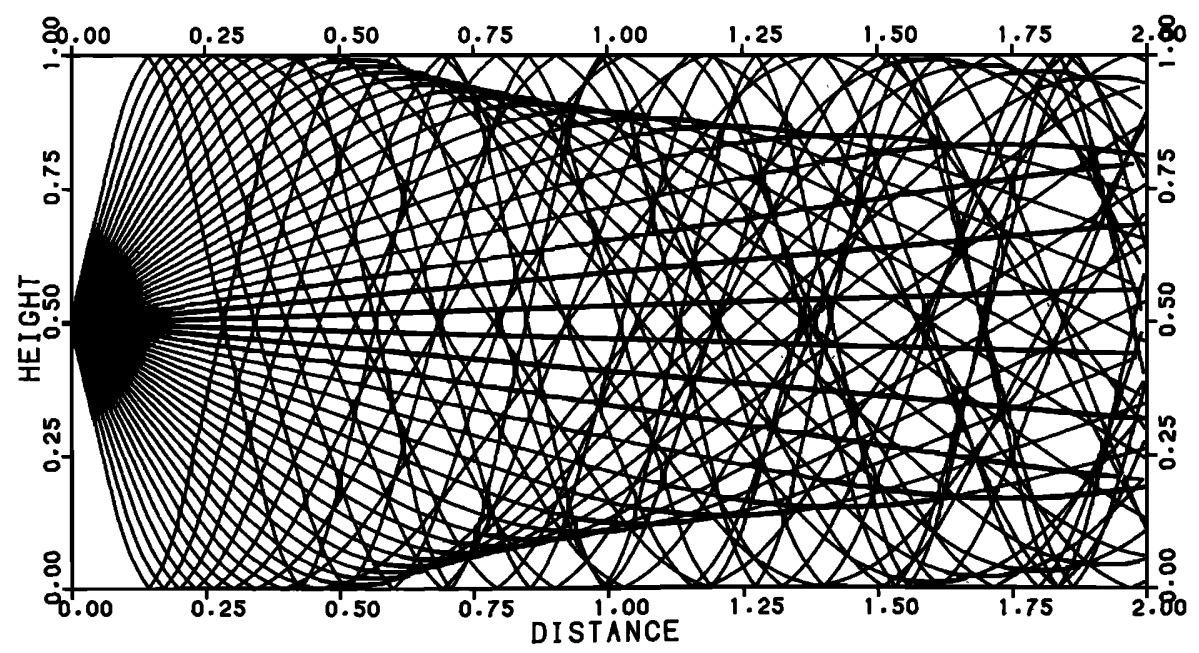

FIG. 4. Ray diagram for upstream propagation; centerline Mach No. $=0.5$; shear-layer thickness of $30 \%$ of the duct height. 


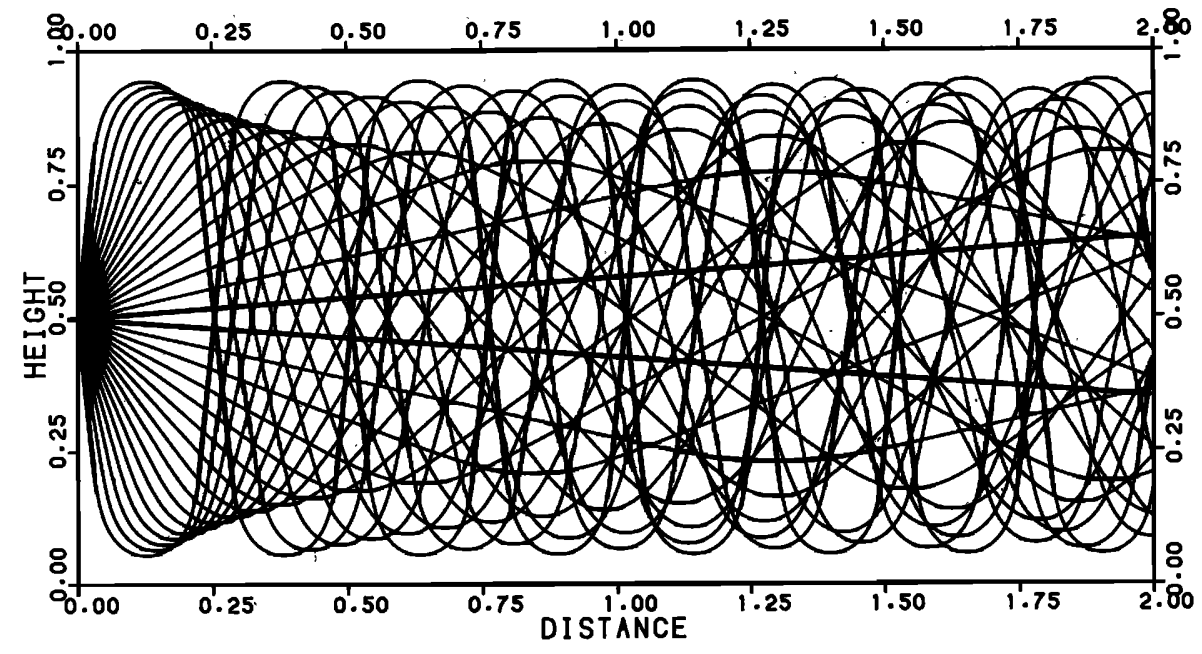

FIG. 5. Ray diagram for upstream propagation; centerline Mach No. $=0.7$; shear-layer thickness of $30 \%$ of the duct height.

sound away from the duct walls is evident, as is the dependence on Mach number. The upstream propagation diagrams are also characterized by the presence of caustics. From a purely geometrical point of view, the location of a caustic can be identified as the envelope of points where the ray tube areas go to zero. These can be recognized from the fact that adjacent rays cross without encountering a reflecting surface. Ray methods cannot be used to calculate the sound field on or near these lines. When the ray bundle area shrinks to zero, theory indicates that the intensity would go to infinity. In reality, the intensity near the location of a caustic will not be infinite, but the region will be a region of high energy concentration.

Although the appearance of caustics prevented calculating the intensity in the duct for upstream propagation, the ray plots provide a good indication of where the acoustic energy should be concentrated. The highest concentration of energy should occur in the shear layers near the caustics. In the central part of the duct, the intensity should vary widely because of the interference between the reflected and turned rays. In the regions where there are no rays adjacent to the duct walls, ray theory predicts a shadow zone with complete silence. Higher-order solutions have shown, however, that in such regions the sound field decreases monotonically with distance from the maximum near a caustic.
Figures 6-9 show the results obtained for downstream propagation. In the first three figures (Figs. $6-8$ ), the shear-layer thickness has been held at $30 \%$ of the duct height, while the centerline Mach number has been varied from 0.3 to 0.7. For Figs. 7 and 9 , however, the centerline Mach number has been held at $\mathbf{0 . 5}$ and the shear-layer thickness varied from $30 \%$ to $10 \%$ of the duct height.

Each of the ray plots for downstream propagation is similar in appearance. Unlike the upstream ray plots, the bending of the rays was not as pronounced in the shear layers. The rays bend slightly towards the duct wall in the shear-layer region. Less bending was observed for the downstream case because the flow gradients did not contribute to variations in the index of refraction to the degree present in upstream propagation. This was because the index of refraction $\mu$ is related to both the flow Mach number and the cosine of the angle $\theta$ between the wave normal and the flow velocity direction by the relationship

$$
\mu=[1+M(z) \cos \theta]^{-1} \text {. }
$$

For the higher downstream angles of incidence, the component of flow velocity in the direction of propagation was very small. Thus variations in the flow velocity had small effects on the wave phase velocity. For upstream propagation, the rays were bent in the direc-

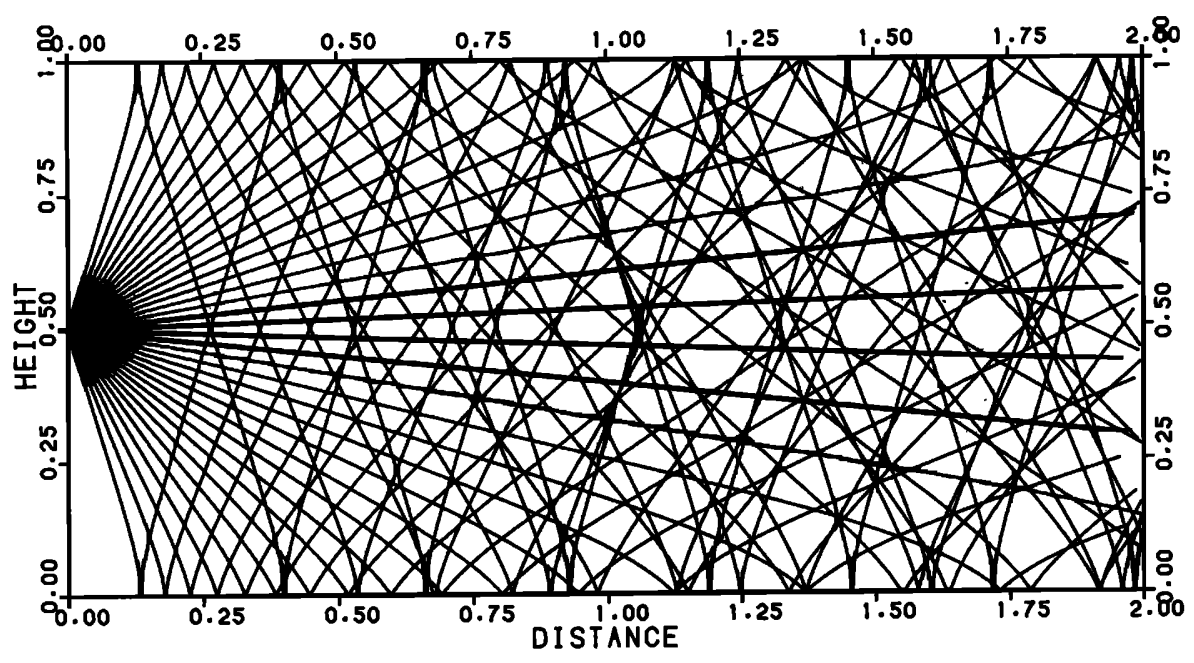

FIG. 6. Ray diagram for downstream propagation; centerline Mach No. $=\mathbf{0 . 3}$; shear-layer thickness of $30 \%$ of the duct height. 


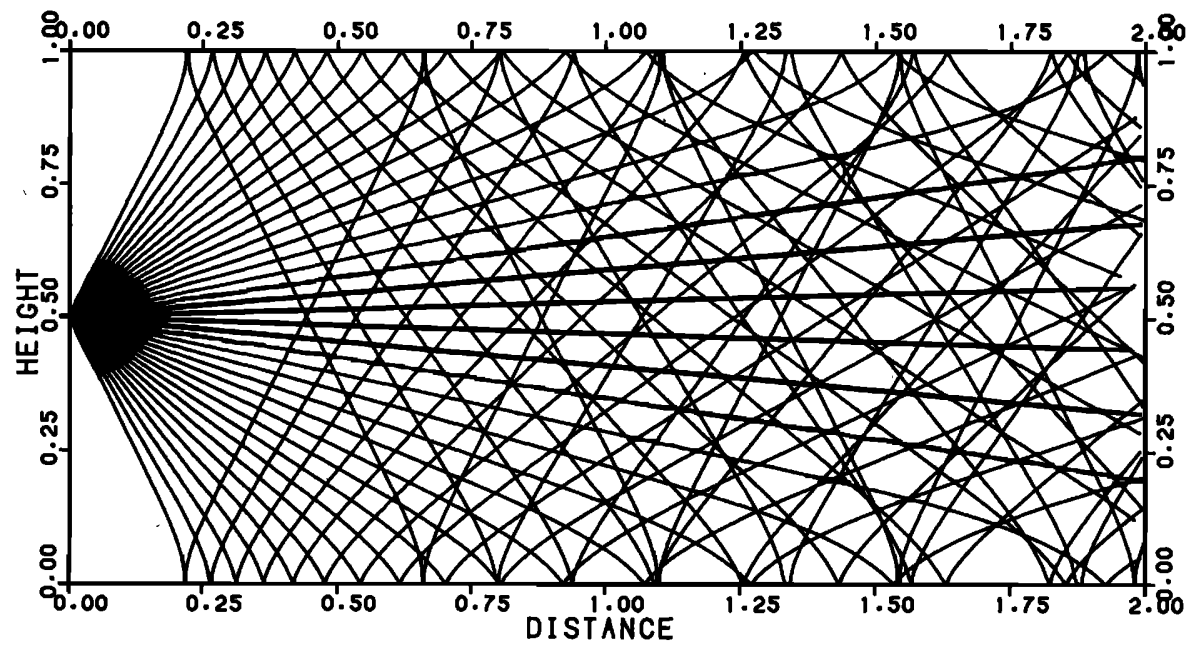

FIG. 7. Ray diagram for downstream propagation; centerline Mach No. $=0.5$; shear-layer thickness of $30 \%$ of the duct height. tion of decreasing wave normal angles to the point where some rays even reached turning points. For these low angles of incidence the contribution of the flow velocity component to the phase velocity was maximum. Flow gradients caused greater changes in the phase velocity and thus caused greater refraction of the rays.

The phase velocity for the downstream rays was found to be greater than the phase velocity for the upstream rays. This result can be attributed to the component of flow in the direction of wave propagation. The ray velocity was also greatest for downstream propagation. The ray velocity has a significant effect on the distribution of energy in the duct, because energy flow is found from the product of intensity and ray velocity. Therefore, the total energy which flows through a given point in an instant of time is a function of both the intensity of the ray and the speed of energy transport along the ray.

\section{INTENSITY LOSS CALCULATIONS}

The ray diagrams yield interesting information about the paths of propagation of the acoustic energy. However, it is also important to know the intensity of the sound at various locations in the duct. Consequently, the next step is to determine the intensity profiles across the duct. This was done in terms of the loss of intensity from the reference intensity close to the source.

The intensity $I_{1}$ at any point along a ray path can be related to a reference intensity $I_{0}$ at a point near the sound source by the basic energy relationships

$$
I_{0} A_{0}=I_{1} A_{1} \text {, }
$$

where $A_{0}$ and $A_{1}$ are ray-bundle areas. Spreading loss accounts for the changes in intensity associated with the changes in ray-bundle area along the ray path, and it can be calculated directly from ray geometry. The spreading loss of a ray bundle is defined as

$$
N=-10 \log \left(I / I_{0}\right)=-10 \log \left(A_{0} / A\right) \mathrm{dB} .
$$

Thus the problem of calculating the intensity loss along a ray path becomes a geometric problem stated in terms of ray-tube area.

The set of differential equations required to calculate spreading loss were derived through partial differentiation of the ray-trace equations. This differentiation was performed with respect to $\theta_{0}$, the initial angle of the wave normal. The approach follows one developed by Anderson et al. ${ }^{18}$ and by Ugincius, ${ }^{19}$ for an inhomogeneous still medium. The approach was applied here to a homogeneous moving medium.

Consider two rays separated by a small initial angle

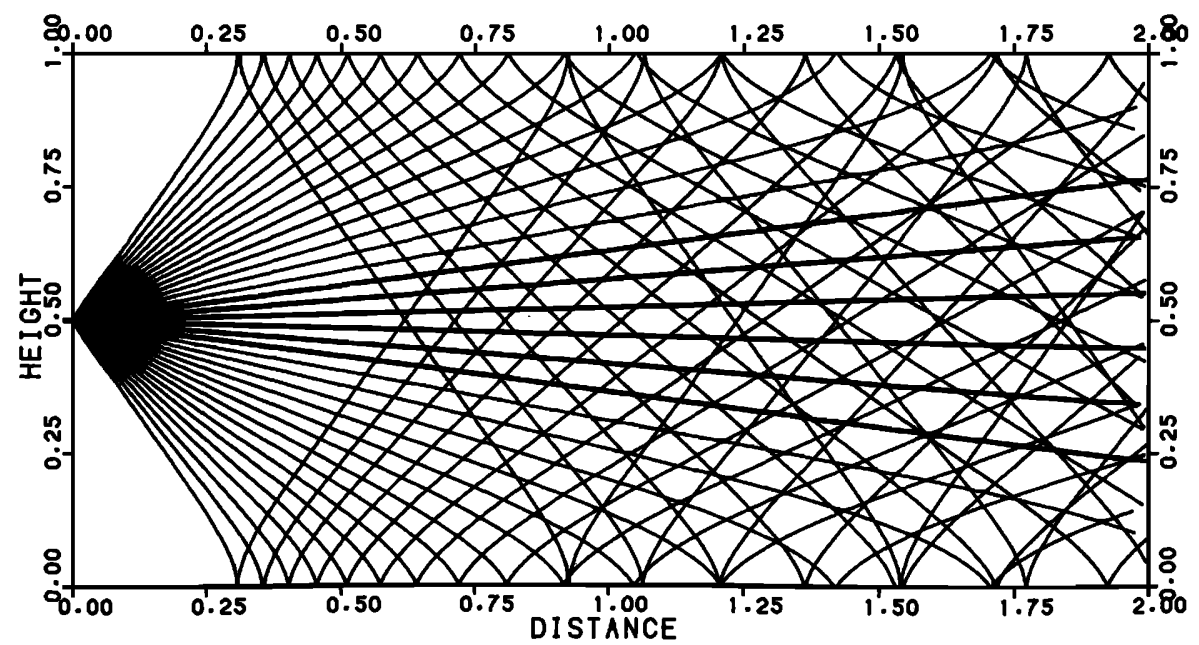

FIG. 8. Ray diagram for downstream propagation; centerline Mach No. $=0.7$; shear-layer thickness of $30 \%$ of the duct height. 


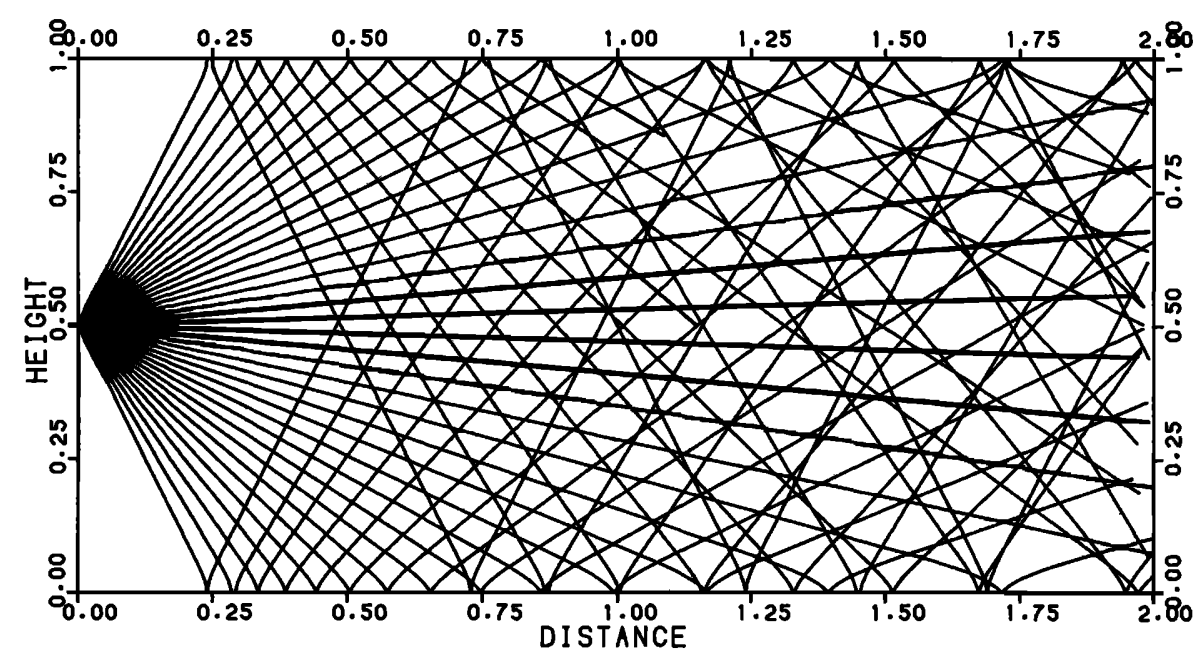

FIG. 9. Ray diagram for downstream propagation; centerline Mach No. 0.5; shear-layer thickness of $10 \%$ of the duct height. difference as shown in Fig. 10. It is assumed that the intensity is known at a reference radial distance $r_{0}$ from the source, and that all spreading losses or gains may be calculated from this point. The ray-bundle area at this radius for a unit-width slice of the wavefront will be

$$
d A_{0}=r_{0} d \theta_{0} .
$$

Now consider points $A$ and $B$ which lie on adjacent rays at points of equal phase. To calculate the raybundle area, it is necessary to calculate the arc length of the wavefront's $x z$-plane intercept connecting these points. At points of equal time, the location of points $A$ and $B$ become functions of another independent variable besides time, the initial wave normal angle $\theta_{0}$. For rays distinguished by a small initial angle change, points of equal phase will be separated in relation to the derivative of the ray-trace variables $(x, z)$ with respect to the initial wave normal angle. This gives rise to a set of spreading loss variables which are defined as follows:

$$
X_{t}=\left(\frac{d x^{*}}{d \theta_{0}}\right)_{t}, \quad Z_{t}=\left(\frac{d z^{*}}{d \theta_{0}}\right)_{t}, \quad \Theta_{t}=\left(\frac{d \theta}{d \theta_{0}}\right)_{t} .
$$

Another variable closely related to these is the corresponding change in arc length of the wavefront with respect to $\theta_{0}$ which is symbolized by

$$
W_{t}=\left(\frac{d w}{d \theta_{0}}\right)_{t}
$$

where $W=$ ray bundle arc length. From the geometry shown in Fig. 10, this arc length variable can be written in terms of the variable $Z$ as

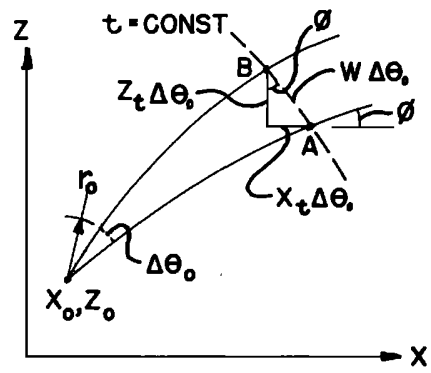

FIG. 10. Side view of an infinitesimal ray bundle.

$$
W_{t}=Z_{t} / \cos \theta_{0} \text {. }
$$

When the solution of Eq. (17) is known, the ray-tube area for a unit slice of the wave can be written as

$$
d A=W d \theta_{0} \text {. }
$$

The spreading loss along the ray path can then be calculated by the use of Eq. (12) as

$$
N=-10 \log \left(\frac{d A_{0}}{d A}\right)=-10 \log \left(\frac{r_{0}}{W}\right) \text {. }
$$

The procedure used for calculating spreading loss, required solving for the variables defined by Eq. (15). Differentiation of the ray-trace system of equations, with respect to $\theta_{0}$ yields the following:

$$
\begin{aligned}
& \frac{d X}{d T^{*}}=-\sin \theta \Theta_{t}+\frac{d M(z)}{d z} Z_{t}, \\
& \frac{d Z}{d T^{*}}=\cos \theta \Theta_{t}, \\
& \frac{d \Theta}{d T^{*}}=\frac{-d^{2} M\left(z^{*}\right)}{d^{2} z^{*}} Z_{t} \cos ^{2} \theta+2 \frac{d M\left(z^{*}\right)}{d z^{*}} \cos \theta \sin \theta \Theta_{t} .
\end{aligned}
$$

The initial conditions can be calculated by evaluating $x^{*}\left(T^{*}\right), y^{*}\left(T^{*}\right)$, and $\theta\left(T^{*}\right)$ at $T^{*}=0$ and then differentiating with respect to $\theta_{0}$. Performing this differentiation gives the required initial conditions for the spreading loss system:

$$
X_{t}(0)=0, \quad Z_{t}(0)=0, \quad \Theta_{t}(0)=1 .
$$

The solutions to the spreading loss equations [(20a)$(20 \mathrm{c})$ ], along with the ray-trace equations, furnished enough information to calculate spreading loss at any point on a given ray for a boundless medium with a continuous index of refraction.

Since the transmitting medium in this case is not infinite, however, it is necessary to find the boundary conditions using the same variables used in the spreading loss calculations. This derivation has been reported by Ugincius. ${ }^{19}$ Ugincius used length along the rays as his independent parameter, rather than time, $t$, as was used in this development. However, the spreading loss is a scalar point function and is thus independent of the 
ray parameters, as was proved by Warfield and Jacobson..$^{20}$ Similarly, the reflection conditions can be derived from first principles and shown to be independent of the ray parameters (see Grimm). ${ }^{21}$ It should be noted that when time, rather than distance along the ray, is used, the necessary derivative in the numerator of the second term of Eq. (22c) is $d \theta / d T^{*}$, not $d \theta / d s$, so that the substitution of ray curvature made by Ugincius must not be followed.

Derivation of the boundary conditions begins with recognition that the ray parameters may be expressed as functions of $x$ and $z$, which in turn are functions of the independent variables time $t$ and starting angle $\theta_{0}$ :

$$
f=f\left[x\left(t, \theta_{0}\right), z\left(t, \theta_{0}\right)\right] .
$$

Formal manipulations with the derivative relationships, as detailed in Ugincius, ${ }^{19}$ yield

$$
\begin{aligned}
& X_{t}=X_{z}+\left(y^{\prime} / z^{\prime}\right) Z_{t}, \\
& Z_{t}=Z_{z}+\left(z^{\prime} / y^{\prime}\right) X_{t}, \\
& \Theta_{t}=\Theta_{z}+\left(\theta^{\prime} / z^{\prime}\right) Z_{t}
\end{aligned}
$$

where the primes indicate differentiation with respect to time $T^{*}$ and capital letters represent differentiation with respect to $\theta_{0}$. The values of all parameters just after reflection can be expressed as the sum of the value before reflection and a change $(\Delta)$ in the value. The changes in the values may be obtained by subtracting the parameter values prior to reflection from those just after reflection. The reflection conditions require

$$
\begin{aligned}
& \Delta x^{\prime}=0, \quad \Delta z^{\prime}=2 z^{\prime}, \\
& \Delta X_{z}=0, \quad \Delta \theta_{z}=-2 \theta_{z} .
\end{aligned}
$$

Furthermore, since the velocity is a function of $z$ only, $d \theta / d T^{*}$ does not change at a horizontal boundary, so

$$
\Delta\left(d \theta / d T^{*}\right)=0
$$

Algebraic combination of the $\Delta$ forms of Eqs. (22) with Eqs. (23) and (24), yield

$$
\begin{aligned}
& \Delta X_{t}=-\left(x^{\prime} / z^{\prime}\right)\left(2 Z_{t}+\Delta Z_{t}\right), \\
& \Delta Z_{t}=-\left[2 Z_{t}+\left(z^{\prime} / y^{\prime}\right) \Delta X_{t}\right], \\
& \Delta \Theta_{t}=-\left[2 \Theta_{t}+\left(\theta^{\prime} / z^{\prime}\right) \Delta Z_{t}\right] .
\end{aligned}
$$

An additional condition is needed to solve this set of equations. With Ugincius, we choose $\Delta X_{t}=0$, since $x^{\prime}$ is a continuous function of $t$ and $\theta_{0}$ at the reflection point. The derivative relationships needed are those of Eqs. (9) and (10). With these substitutions, the boundary conditions become

$$
\begin{aligned}
& \Delta X_{t}=0, \quad \Delta Z_{t}=-2 Z_{t}, \\
& \Delta \Theta_{t}=2\left(\frac{-\cos ^{2} \theta[(d M(z) / d z)] Z_{t}}{\sin \theta}-\Theta_{t}\right) .
\end{aligned}
$$

These are the necessary boundary conditions. The spreading loss equations could then be solved using a Runge-Kutta integration scheme.

Spreading loss profiles at a selected duct location were obtained by finding the spreading loss at 97 of 100 equally spaced points across the duct at the desired $x$ location. Finding the spreading loss at any one point involved finding the spreading loss along each of the rays emanating from the sound source and passing through the point. A coherent summation of the spreading loss at the point could then be obtained. In theory this summation would have to carried out over an infinite number of rays passing through the source and receiver points. In practice, however, it was found that finding the five shortest rays was sufficient. Inclusion of additional (longer) rays did not change the intensity loss calculations appreciably.

Finding the five shortest ray paths was a major numerical calculation problem. The difficulty arose in trying to constrain the ray-trace solution to pass through a fixed end point at some unknown time along the ray path. An iterative scheme relying on complete calculation of the ray path, followed by initial angle correction and re-calculation, was found to be too time consuming. An approach which proved to be much more efficient was the ray sweep-out method. ${ }^{22}$ Since the phase velocity varied only with height, it was possible to calculate each ray to its first wall reflection and from that to predict its final position relative to the receiver point. A Newton-Raphson scheme was then used to adjust the initial ray angle so that the next ray calculated would pass through the desired receiver point.

An arbitrary axial distance equal to one duct height $\left(x^{*}=1\right)$ was chosen for summing the contributions from the rays. The arbitrary reference distance $r_{0}$ in Eq. (19) was chosen as 0.05 , for $\frac{1}{20}$ of the duct height. The effect of this choice was simply an overall scaling of the intensity loss without change in the shape of the intensity loss curve.

Typical intensity loss plots are shown in Figs. 11-14. Figures 11 and 12 give the intensity loss profiles for the rays shown in Fig. 7 (centerline Mach number of 0.5 and boundary-layer thickness of $30 \%$ of the duct height). Different frequencies were used for these two cases, however, resulting in different interference effects at the receiver locations. Figure 11 is for a frequency which would have a wavelength of $\frac{1}{10}$ of the duct height, while Fig. 12 is for a frequency which would have a wavelength of $\frac{1}{20}$ of the duct height. Figure 13 shows the effect of changing the boundary-layer thickness: All conditions are the same as for Fig. 12 except for boundary-layer thickness. Figure 14 shows the effect of changing the centerline Mach number on the results. Conditions for Fig. 14 are identical to those for Fig. 12 except for flow rate.

In general, all of these figures show marked intensity level changes as one proceeds across the duct. These changes are the result of constructive or destructive interference between sound travelling along the rays 


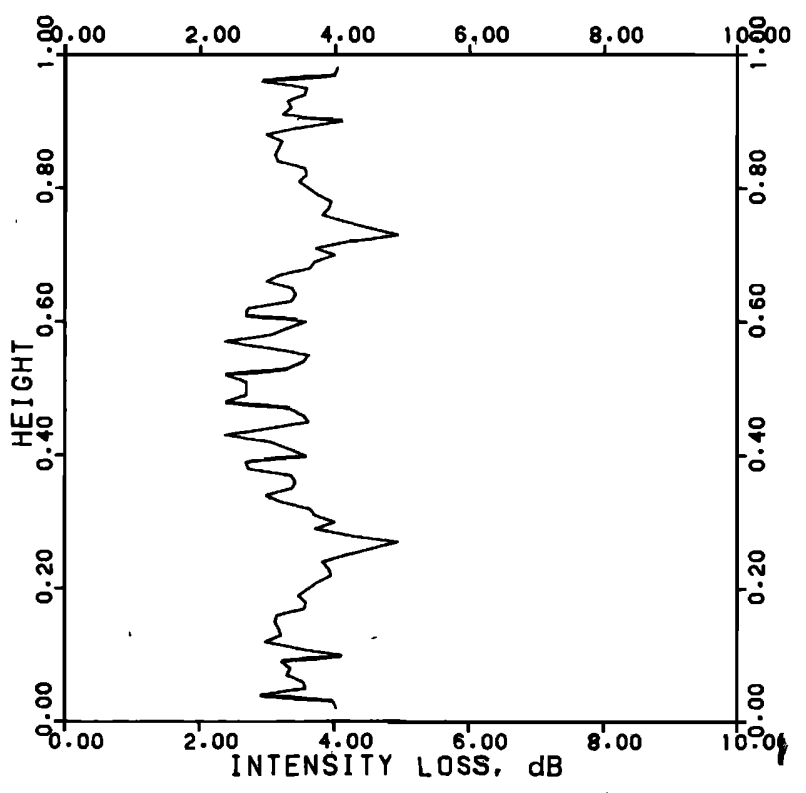

FIG. 11. Downstream intensity loss at a distance of 1 duct height from the source for a centerline Mach No. of 0.5, a shear layer of $30 \%$ of duct height, and a wavelength of $10 \%$ of the duct height.

reaching each point. These results were consistent with the observation, made during debugging of the computer program, that extremely small initial angle changes could often result in great changes in ray destination and path length. This was particularly true for the reflected rays. The number of peaks and valleys in the plot approximately doubled when the frequency was doubled, since the sensitivity of intensity loss to path length changes was doubled. Two intensity loss

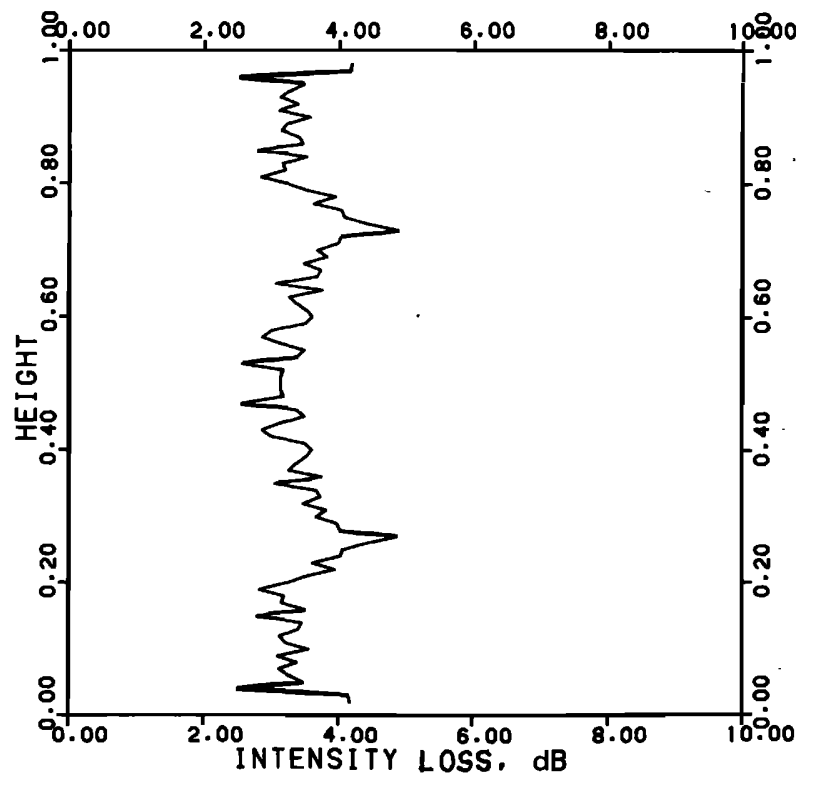

FIG. 12. Downstream intensity loss at a distance of 1 duct height from the source, at a centerline Mach No. of 0.5, a shear-layer thickness of $30 \%$ of duct height, and a wavelength of $5 \%$ of the duct height.

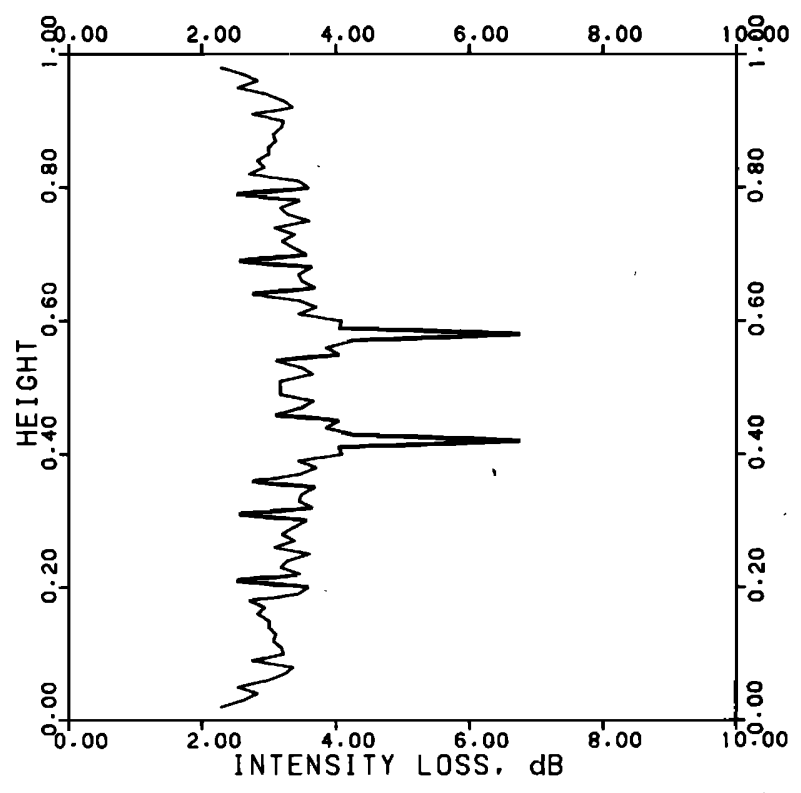

FIG. 13. Downstream intensity loss at a distance of 1 duct height from the source, at a centerline Mach No. of 0.5, a shear-layer thickness of $10 \%$ of duct height, and a wavelength of $5 \%$ of the duct height.

peaks appeared in each plot, and these can be seen to change position markedly with changes in flow parameters. When a centerline Mach number of 0.7 was used, the diffraction effects became quite strong. It is interesting to note on that plot the appearance of negative intensity loss spikes, indicating that the intensity at those heights one duct height downstream was greater than the intensity at a distance of 0.05 from the source.

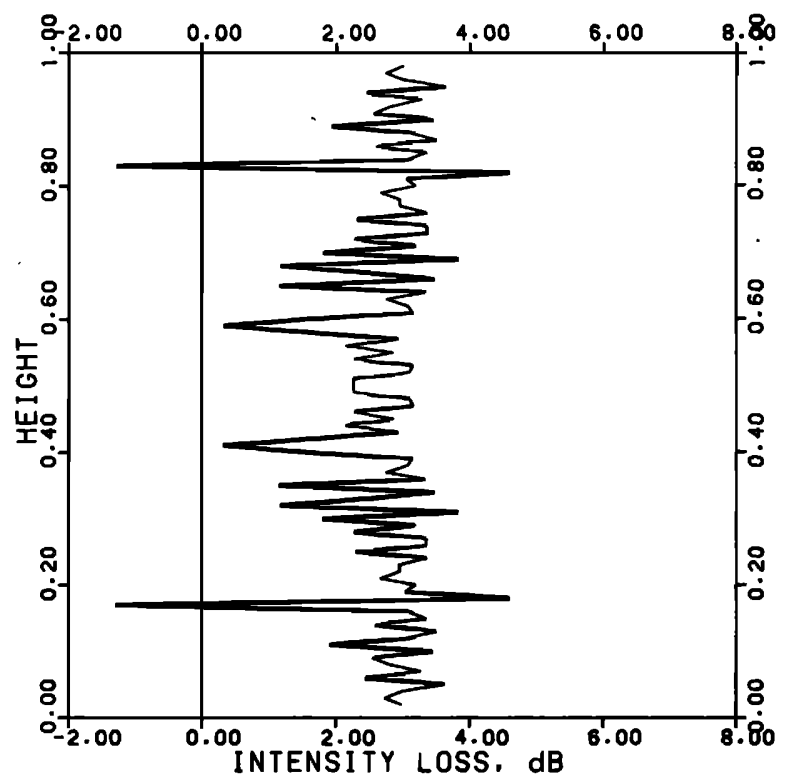

FIG. 14. Downstream intensity loss at a distance of 1 duct height from the source, at a centerline Mach No. of 0.7 , a shear-layer thickness of $30 \%$ of duct height, and a wavelength of $5 \%$ of the duct height. 


\section{CONCLUSIONS}

Geometric acoustics was found to be a valuable tool with which to study the propagation of sound through a sheared flow within a hard-walled duct. It was found that the ray plots could provide an excellent representation of the sound field by showing the paths of energy flow, and that the intensity loss plots could be used to show the distribution of acoustic energy at a specified cross section of a duct. The method was applied to a variety of flow velocity and flow-gradient situations. The method encountered difficulty in upstream propagation, where the theory broke down because of the appearance of caustics.

Two phenomena were found which affected the sound field as a result of the flow of the medium. First, the effects of refraction were found to result from variations in the wave phase velocity encountered as the sound propagated through regions of changing flow velocity. These effects were found to be greater for upstream propagation where the rays bent very sharply towards the center of the duct, and less significant for downstream propagation where the rays were bent only slightly towards the walls of the duct. Second, convective effects of the flow were shown to cause the wave to travel faster for downstream propagation and slower for upstream propagation.

The geometric acoustics theory proved to be simpler to apply than normal-mode theory for the case with flow in the duct. Further extensions of this method to include finite boundary impedances and variable duct geometry also appear simple, and open the possibility of obtaining solutions to acoustic problems which are not yet tractable with normal-mode approaches.

${ }^{1} \mathrm{U}$. Ingard, "Influence of Fluid Motion Past a Plane Boundary on Sound Reflection, Absorption, and Transmission," J. Acoust. Soc. Am. 31, 1035-1036 (1959).

${ }^{2} \mathrm{~W}$. Eversman, "The Effect of Mach Number on the Tuning of an Acoustic Lining in a Flow Duct," J. Acoust. Soc. Am. 48, 425-428 (1970).

${ }^{3}$ C. L. Morfey, "Rotating Pressure Patterns in Ducts: Their Generation and Transmission," J. Sound Vib. 1, 60-87 (1964).

${ }^{4}$ D. H. Tack and R. F. Lambert, "Influence of Shear Flow on Sound Attenuation in Lined Ducts," J. Acoust. Soc. Am. 38, 655-666 (1965).
${ }^{5}$ P. E. Doak and P. G. Vaidya, "Attenuation of Plane Wave and Higher Order Mode Sound Propagation in Lines Ducts," J. Sound Vib. 12, 201-224 (1970).

${ }^{6}$ D. C. Pridmore-Brown, "Sound Propagation in a Fluid Flowing Through an Attenuating Duct," J. Fluid Mech. 4, 393406 (1958).

${ }^{7}$ P. Mungar and G. M. L. Gladwell, "Acoustic Wave Propagation in a Sheared Fluid Contained in a Duct," J. Sound Vib. 9, 28-48 (1969).

${ }^{8}$ F. Mechel, P. Mertens, and W. Schilz, "Research on Sound Propagation in Sound-Absorbent Ducts with Superimposed Air Streams," AMRL Rep. No. TDR-62-140, III, Wright Patterson Air Force, Ohio, December 1962.

${ }^{9}$ P. Mungar and H. E. Plumblee, "Propagation and Attenuation of Sound in a Soft-Walled Annular Duct Containing a Sheared Flow," Presented at the NASA Basic Noise Research Conference on July 14-15, 1969.

${ }^{10}$ W. Eversman, "Effect of Boundary-Layer on the Transmission and Attenuation of Sound in an Acoustically Treated Circular Duct," J. Acoust. Soc. Am. 49, 1372-1380 (1971).

${ }^{11}$ A. S. Hersh and I. Catton, "Effect of Shear Flow on Sound Propagation in Rectangular Ducts," (A. S. Hersh, Bolt Beranek and Newman Inc., CA, 1970).

${ }^{12}$ S. D. Savkar, "Propagation of Sound in Ducts with Shear Flow," J. Acoust. Soc. Am. 19, p. 355-372 (1971).

${ }^{13}$ A. H. Nayfeh, J. E. Kaiser, and D. P. Telionis, "Acoustics of Aircraft Engine-duct Systems," AIAA J. 13, 130-153 (1975).

${ }^{14}$ D. W. Grimm, Application of Ray Acoustics to the Propagation of Sound in Ducts Containing Sheared Flows, unpublished M. S. thesis, Virginia Polytechnic Institute and State University, 1972.

${ }^{15}$ P. Mungar and H. E. Plumbee, "Propagation and Attenuation of Sound in a Soft-Walled Annular Duct Containing a Sheared Flow," presented at the NASA Basic Noise Research Conference on 14-15 July 1969.

${ }^{16}$ See. Ref. 11.

${ }^{17}$ W. D. Hayes, R. C. Haefeli, and H. E. Kulsrud, "Sonic Boom Propagation in a Stratified Medium with Computer Program," NASA CR-1229, April 1969.

${ }^{18}$ G. Anderson, R. Gocht, and D. Sirota, J. Acoust. Soc. Am. 36, 140-145 (1964).

${ }^{19} \mathrm{P}$. Ugincius, "Intensity Equations in Ray Acoustics. I," J. Acoust. Soc. Am. 45, 193-205 (1969).

${ }^{20} \mathrm{~J}$. T. Warfield and M. J. Jacobson, "Invariance of Geometric Spreading Loss with Changes in Ray Parametrization," J. Acoust. Soc. Am. 50, 342-347 (1971).

${ }^{21} \mathrm{D}$. W Grimm, Application of Ray Acoustics to the Propagation of Sound in Ducts Containing Sheared Flows, unpublished M. S. thesis, Virginia Polytechnic Institute and State University, 1972.

${ }^{22}$ H. P. Bucker, “The Ray Sweep-Out Method," J. Acoust. Soc. Am. 52, 283-286 (1972). 